\title{
Abstract polymer models with general pair interactions
}

\author{
Aldo Procacci \\ Dep. Matemática-ICEx, UFMG, CP 702 Belo Horizonte - MG, 30161-970 Brazil \\ email: aldo@mat.ufmg.br
}

\begin{abstract}
A convergence criterion of cluster expansion is presented in the case of an abstract polymer system with general pair interactions (i.e. not necessarily hard core or repulsive). As a concrete example, the low temperature disordered phase of the BEG model with infinite range interactions, decaying polynomially as $1 / r^{d+\lambda}$ with $\lambda>0$, is studied.
\end{abstract}

\section{Introduction}

The abstract polymer gas is an important tool to study the high temperature/low density or low temperature phase of many statistical mechanics models. Generally speaking, the abstract polymer model consists of a collection of objects (the polymers) which play the role of the particles of the gas. These polymers have a given activity and they interact via a hard core pair potential suitably defined. Typically, one wants to show that the pressure of this polymer gas can be written in terms of an absolutely convergent series if the activities are taken sufficiently small.

The first example of such a model appeared in [9] where the polymers were finite non overlapping subsets of the cubic lattice $\mathbb{Z}^{d}$. The authors proved convergence of the pressure via the method of Kirkwood-Salsburg equations. Subsequently, the same system studied in [9] was treated in [18] and [5] via cluster expansion methods based on tree graph inequalities.

In [10] the most general version of this system was given. There, polymers were simply a collection of objects with a given activity and interacting through an hard core pair potential introduced via a symmetric and reflexive relation in the polymer space. Polymers belonging to this relation were called incompatible, and compatible otherwise. The hard core condition was simply to forbid configurations of polymers containing pairs of incompatible polymers. Differently from the cases considered previously, in which polymers had a cardinality and a size, the Kotecký-Preiss polymers were characterized only by the activity.

In [6] the convergence condition for the Kotecký-Preiss polymer gas was slightly improved and the proof was greatly simplified, being reduced to a simple inductive argument, as it was shown very clearly in [12] and [19]. In particular, in [19] it has been observed that the Dobrsushin's proof works even for more general abstract polymer gases, in which polymers may interact through a repulsive soft-core pair interaction.

Very recently [8] the Kotecký-Preiss and the Dobrushin conditions for convergence of the abstract polymer gas with purely hard core interactions were reobtained via the standard cluster expansion methods and a new improved condition was given by exploiting an old tree graph identity valid for hard core systems due to O. Penrose [14]. 
In all these works, the basically hard core character of the interaction seemed to be an essential ingredient to control the convergence. Exceptions can be found in [7, [1]. In [7] a contour model with interaction (exponentially decaying al large distances) is proposed. However the model is rewritten in term of the usual hard core polymer gas where polymers are objects more complicated than the original contours. This philosophy has also been pursued in [11] where a one-dimensional contour model with long range interaction is rewritten in term of new objects with hard core pair interactions.

It would be of interest to treat also cases in which polymers interact via more general pair interactions, e.g., not necessarily repulsive, not necessarily hard core, not necessarily finite range. Such abstract polymer model could be a useful tool in the study of spin systems at low temperature interacting via infinite range polynomially decaying potential, see e.g. [13].

In this paper we develop a model of abstract polymers (of the type of [10]) with interactions more general than the hard-core. Our polymers interact through a "short distance" repulsive (not necessarily hard core) pair potential which is non zero only on pair of incompatible polymers, plus an a pair potential with no definite sign (hence it can be attractive), acting only on pairs of compatible polymers. We give a condition convergence for the pressure of this gas by using a cluster expansion method similar to the one developed in [8]. However, differently from [8], we could not use here the Penrose identity, since our interaction is not purely hard-core. We rather used another well known tree graph identity originally proposed in [3] and further developed in [1].

The rest of the paper is organized as follows. In section 2 we introduce the model, notations and the main result of the paper. In section 3 we give the proof of our result (theorem 1). Namely, in subsection 3.1. we present the tree graph identity and show how it can be used to bound the Ursell coefficients of the Mayer series of our polymer model. In subsection 3.2 we give the convergence argument based on map iterations developed in [8]. In subsection 3.3 we conclude the proof of our main theorem. Finally in section 4, as an example, we use theorem 1 to study the low temperature disordered phase of the BEG model with infinite range interactions with polynomial decay of the type $1 / r^{d+\lambda}$ with $\lambda>0$.

\section{Polymer gas: notations and results}

\subsection{The model.}

Let $\mathcal{P}$ denotes the set of polymers (i.e. $\mathcal{P}$ is the single particle state space). We will assume here that $\mathcal{P}$ is a countable set. We associate to each polymer $\gamma \in \mathcal{P}$ a complex number $z_{\gamma}$ (a positive number in physical situations) which is interpreted as the activity of the polymer $\gamma$. We will denote $z=\left\{z_{\gamma}\right\}_{\gamma \in \mathcal{P}}$.

Polymers interact through a pair potential. Namely, the energy $E$ of a configuration $\gamma_{1}, \ldots, \gamma_{n}$ of $n$ polymers is given by

$$
E\left(\gamma_{1}, \ldots, \gamma_{n}\right)=\sum_{1 \leq i<j \leq n} V\left(\gamma_{i}, \gamma_{j}\right)
$$

where pair potential $V\left(\gamma, \gamma^{\prime}\right)$ is a symmetric function in $\mathcal{P} \times \mathcal{P}$ taking values in $\mathbb{R} \cup\{+\infty\}$. Observe that we don't make any hypothesis on the sign of $V\left(\gamma_{i}, \gamma_{j}\right)$ so this interaction could be for some pairs attractive and for other pairs repulsive.

Fix now a finite set $\Lambda \subset \mathcal{P}$ (the "volume" of the gas). Then the probability to see the configu- 
ration $\left(\gamma_{1}, \ldots, \gamma_{n}\right) \in \Lambda^{n}$ is given by

$$
\operatorname{Prob}\left(\gamma_{1}, \ldots, \gamma_{n}\right)=\frac{1}{\Xi_{\Lambda}} z_{\gamma_{1}} z_{\gamma_{2}} \ldots z_{\gamma_{n}} e^{-\sum_{1 \leq i<j \leq n} V\left(\gamma_{i}, \gamma_{j}\right)}
$$

where the normalization constant $\Xi_{\Lambda}$ is the grand-canonical partition function in the volume $\Lambda$ and is given by

$$
\Xi_{\Lambda}(z)=1+\sum_{n \geq 1} \frac{1}{n !} \sum_{\left(\gamma_{1}, \ldots, \gamma_{n}\right) \subset \Lambda^{n}} z_{\gamma_{1}} z_{\gamma_{2}} \ldots z_{\gamma_{n}} e^{-\sum_{1 \leq i<j \leq n} V\left(\gamma_{i}, \gamma_{j}\right)}
$$

Note that the configurations $\gamma_{1}, \ldots, \gamma_{n}$ for there exist a pair $\gamma_{i}, \gamma_{j}$ such that $V\left(\gamma_{i}, \gamma_{j}\right)=+\infty$ have zero probability to occur, i.e. are forbidden. So, following the tradition, if a pair $\left(\gamma, \gamma^{\prime}\right) \in \mathcal{P} \times \mathcal{P}$ is such that $V\left(\gamma, \gamma^{\prime}\right)=+\infty$, we will denote by $\gamma \not \gamma^{\prime}$ and say that $\gamma$ and $\gamma^{\prime}$ are incompatible.

Since we are admitting non purely repulsive interaction among polymers, we also need to require that the potential energy $E$ is stable in the classical sense. This can be achieved by imposing that there exists a function $B(\gamma) \geq 0$ such that

$$
\sum_{1 \leq i<j \leq n} V\left(\gamma_{i}, \gamma_{j}\right) \geq-\sum_{i=1}^{n} B\left(\gamma_{i}\right)
$$

for all $n \in \mathbb{N}$ and all $\left(\gamma_{1}, \ldots, \gamma_{n}\right) \in \mathcal{P}^{n}$. Note that in the case in which $\left(\gamma_{1}, \ldots, \gamma_{n}\right)$ contains pairs of incompatible polymers the l.h.s. of $(2.3)$ is $+\infty$ so this inequelity is trivially satisfied.

The stabiltity condition immediately implies that $\Xi_{\Lambda}$ is convergent and

$$
\Xi_{\Lambda} \leq 1+\sum_{n \geq 1} \frac{1}{n !}\left[\sum_{\gamma \subset \Lambda} z_{\gamma} e^{B(\gamma)}\right]^{n} \leq \exp \left\{\sum_{\gamma \in \Lambda} z_{\gamma} e^{B(\gamma)}\right\} \leq|\Lambda| \max _{\gamma \in \Lambda} \exp \left\{z_{\gamma} e^{B(\gamma)}\right\}
$$

Actually, (2.3) implies that $\Xi_{\Lambda}(z)$ is analytic in the whole $\mathbb{C}^{|\Lambda|}(|\Lambda|$ is the cardinality of $\Lambda)$.

As we said in the introduction, the usual choice available in the literature is that $V\left(\gamma, \gamma^{\prime}\right)$ takes values in the set $\{0,+\infty\}$ for all $\left(\gamma, \gamma^{\prime}\right) \in \mathcal{P} \times \mathcal{P}$ and $V(\gamma, \gamma)=+\infty$ for all $\gamma \in \mathcal{P}$ (purely hard core pair potential) but we remark that the purely repulsive case (i.e. $0 \leq V\left(\gamma_{i}, \gamma_{j}\right) \leq+\infty$ for all pairs) has also been considered in [19] and [20. However, in view of the possible connections with the low temperature phase of spin systems with infinite range interactions, we think that the most interesting situation treated in the present paper is the case $V\left(\gamma_{i}, \gamma_{j}\right)<0$, i.e. when an attractive potential, possibly infinite range, is acting among polymers.

\subsection{Results.}

The pressure of this gas, namely $\log \Xi_{\Lambda}$, can be written as a formal series through a Mayer expansion

on the Gibbs factor $\exp \left\{-\sum_{1 \leq i<j \leq n} V\left(\gamma_{i}, \gamma_{j}\right)\right\}$. Namely, a standard calculations (see e.g. [5]) gives

$$
\log \Xi_{\Lambda}(z)=\sum_{n=1}^{\infty} \frac{1}{n !} \sum_{\left(\gamma_{1}, \ldots, \gamma_{n}\right) \subset \Lambda^{n}} \phi^{T}\left(\gamma_{1}, \ldots, \gamma_{n}\right) z_{\gamma_{1}} \ldots z_{\gamma_{n}}
$$

with

$$
\phi^{T}\left(\gamma_{1}, \ldots, \gamma_{n}\right)= \begin{cases}1 & \text { if } n=1 \\ \sum_{g \in G_{n}} \prod_{\{i, j\} \in E_{g}}\left(e^{-V\left(\gamma_{i}, \gamma_{j}\right)}-1\right) & \text { if } n \geq 2\end{cases}
$$


where $G_{n}$ is the set all connected graphs with vertex set $\{1,2, \ldots, n\}$. We recall that a graph $g \in G_{n}$ is a pair $g=\left(V_{g}, E_{g}\right)$ where $V_{g}=\{1,2, \ldots, n\}$ is the set of vertices of $g$ and $E_{g} \subset\{\{i, j\} \subset$ $\{1,2, \ldots, n\}\}$ is the set of edges of $g$. We also recall that $g=\left(V_{g}, E_{g}\right)$ is connected if for any $A, B$ such that $A \cup B=V_{g}$ and $A \cap B=\emptyset$, there exists $\{i, j\} \in E_{g}$ such That $A \cap\{i, j\} \neq \emptyset$ and $B \cap\{i, j\} \neq \emptyset$.

The equation (2.4) makes sense only for those $z$ for which the formal series in the r.h.s. of (2.4) converges absolutely. To study absolute convergence, we will consider the positive term series

$$
\left|\log \Xi_{\Lambda}\right|(\rho)=\sum_{n=1}^{\infty} \frac{1}{n !} \sum_{\left(\gamma_{1}, \ldots, \gamma_{n}\right) \subset \Lambda^{n}}\left|\phi^{T}\left(\gamma_{1}, \ldots, \gamma_{n}\right)\right| \rho_{\gamma_{1}} \cdots \rho_{\gamma_{n}}
$$

where now $\rho_{\gamma} \in[0,+\infty)$, for all $\gamma \in \mathcal{P}$ and $\rho=\left\{\rho_{\gamma}\right\}_{\gamma \in \mathcal{P}}$. Of course $\left|\log \Xi_{\Lambda}(z)\right| \leq\left|\log \Xi_{\Lambda}\right|(\rho)$ for $z$ in the polydisk $\left\{\left|z_{\gamma}\right| \leq \rho_{\gamma}\right\}_{\gamma \in \mathcal{P}}$.

We further define, for each $\gamma_{0} \in \mathcal{P}$, a function $\Pi_{\mathcal{P}}^{\gamma_{0}}(\rho)$ directly related to (2.6) (a "pinned" sum defined in the whole set $\mathcal{P}$ ) as follows

$$
\Pi_{\mathcal{P}}^{\gamma_{0}}(\rho)=\sum_{n=0}^{\infty} \frac{1}{n !} \sum_{\left(\gamma_{1}, \gamma_{2}, \ldots, \gamma_{n}\right) \in \mathcal{P}^{n}}\left|\phi^{T}\left(\gamma_{0}, \gamma_{1}, \ldots, \gamma_{n}\right)\right| \rho_{\gamma_{1}} \ldots \rho_{\gamma_{n}}
$$

Clearly, if we are able to show that $\Pi_{\mathcal{P}}^{\gamma_{0}}(\rho)$ converges, then $\left|\log \Xi_{\Lambda}\right|(\rho)$ and hence $\left|\log \Xi_{\Lambda}(z)\right|$ for $\left|z_{\gamma}\right| \leq \rho_{\gamma}$ also converge, since it is easy to check that

$$
\left|\log \Xi_{\Lambda}\right|(\rho) \leq|\Lambda| \sup _{\gamma_{0} \in \Lambda} \rho_{\gamma_{0}} \Pi_{\mathcal{P}}^{\gamma_{0}}(\rho)
$$

To understand the meaning of the series $\Pi_{\mathcal{P}}^{\gamma_{0}}(z)$ just observe that its finite volume version $\Pi_{\mathcal{P}}^{\gamma_{0}}(\rho)$, namely

$$
\Pi_{\Lambda}^{\gamma_{0}}(\rho)=\sum_{n=0}^{\infty} \frac{1}{n !} \sum_{\left(\gamma_{1}, \gamma_{2}, \ldots, \gamma_{n}\right) \in \Lambda^{n}}\left|\phi^{T}\left(\gamma_{0}, \gamma_{1}, \ldots, \gamma_{n}\right)\right| \rho_{\gamma_{1}} \ldots \rho_{\gamma_{n}}
$$

is directly related to $\log \Xi_{\Lambda}\left(\rho_{\Lambda}\right)$. It is immediate to see that

$$
\Pi_{\Lambda}^{\gamma_{0}}(\rho)=\frac{\partial}{\partial \rho_{\gamma_{0}}}\left|\log \Xi_{\Lambda}\right|(\rho)
$$

The main result of the paper is a convergence criterion for the positive series (2.7). Such criterion can be considered as a generalization of the Kotecký-Preiss criterion for polymer system interacting through a pair potential which is not purely hard core. The criterion can be stated as the following theorem.

Theorem 1 . Let $\mu: \mathcal{P} \rightarrow[0, \infty): \gamma \mapsto \mu_{\gamma}$ be a non negative valued function and let, for each $\gamma \in \mathcal{P}, \rho_{\gamma} \in[0, \infty)$ such that

$$
\rho_{\gamma} e^{B(\gamma)} \leq \mu_{\gamma} e^{-\sum_{\tilde{\gamma} \in \mathcal{P}} F(\gamma, \tilde{\gamma}) \mu_{\tilde{\gamma}}}, \quad \forall \gamma \in \mathcal{P}
$$


where $B(\gamma)$ is the function defined in (2.3) and

$$
F\left(\gamma_{i}, \gamma_{j}\right)= \begin{cases}\left|e^{-V\left(\gamma_{i}, \gamma_{j}\right)}-1\right|=1 & \text { if } \gamma_{i} \nsim \gamma_{j} \\ \left|V\left(\gamma_{i}, \gamma_{j}\right)\right| & \text { otherwise }\end{cases}
$$

Then the series $\Pi_{\gamma_{0}}(\rho)$ [defined in (2.7)] converges and satisfies $\rho_{\gamma_{0}} \Pi_{\gamma_{0}}(\rho) \leq \mu_{\gamma_{0}}$.

Remark. Observe that in the usual case $U$ hard-core one obtains from theorem 1 the usual Kotecký-Preiss condition. We recall however when polymers interact just through a purely repulsive potential, one can do better than (2.11). In particular, for the purely hard core case it has been shown in [8] that the condition (2.11) can be considerably improved by taking advantage of the Penrose tree identity [14, (see also [15], 19] [8]) valid in the case of purely hard core interactions.

\section{Proof of theorem 1.}

The strategy of the proof is quite similar to the one used in [8]. In particular we use here the very same convergence argument for positive series which has been developed in [8]. On the other hand, in the present case we cannot use the Penrose identity in order to bound the Ursell coefficients $\left|\phi^{T}\left(\gamma_{1}, \ldots, \gamma_{n}\right)\right|$, since the pair potential is not purely hard-core (and also not purely repulsive). We will rather make use of another well known "tree graph identity" originally proved in [3] (see also [4, 1, 17, 16]).

\subsection{Tree graph inequality for $\left|\phi^{T}\left(\gamma_{0}, \gamma_{1}, \ldots, \gamma_{n}\right)\right|$}

We state the so called tree graph identity [3], [1], 4] by using the notations of [17] and [16]. We use the short notation $\mathrm{I}_{n}=\{1,2, \ldots, n\}$. A graph $\tau=\left(\mathrm{I}_{n}, E_{\tau}\right) \in G_{n}$ is called a tree if and only if its edge set $E_{\tau}$ has cardinality equal to $n-1$. Let us denote by $T_{n}$ the set of trees with vertex set $\mathrm{I}_{n}$.

In the following whenever $U$ is a finite set, $|U|$ denotes its cardinality.

Lemma 2 (Tree graph identity). Let $V_{i j}$, with $1 \leq i<j \leq n$ be $n(n-1) / 2$ real numbers, then the following identity holds

$$
\sum_{g \in G_{n}} \prod_{\{i, j\} \in E_{g}}\left(e^{-V_{i j}}-1\right)=\sum_{\tau \in T_{n}} \prod_{\{i, j\} \in E_{\tau}}\left(-V_{i j}\right) \int d \mu_{\tau}\left(\mathbf{t}_{n-1}, \mathbf{X}_{n-1}\right) e^{-K\left(\mathbf{X}_{n-1}, \mathbf{t}_{n-1}\right)}
$$

where:

- $\mathbf{t}_{n-1}$ denote a set on $n-1$ interpolating parameters $\mathbf{t}_{n-1} \equiv\left(t_{1}, \ldots, t_{n-1}\right) \in[0,1]^{n-1}$;

- $\mathbf{X}_{n-1}$ denote a set of "increasing" sequences of $n-1$ subsets, $\mathbf{X}_{n-1} \equiv X_{1}, \ldots, X_{n-1}$ such as $\forall i, X_{i} \subset \mathrm{I}_{n}$, we must have $X_{i} \subset X_{i+1},\left|X_{i}\right|=i$ and $X_{1}=\{1\}$.

- $K\left(\mathbf{X}_{n-1}, \mathbf{t}_{n-1}\right)$ is a convex decomposition of the potential, explicitly given by

$$
K\left(\mathbf{X}_{n-1}, \mathbf{t}_{n-1}\right)=\sum_{1 \leq i<j \leq n} t_{1}(\{i, j\}) \ldots t_{n-1}(\{i, j\}) V_{i j}
$$


where

$$
t_{l}(\{i, j\})= \begin{cases}t_{l} \in[0,1] & \text { if } i \in X_{l} \text { and } j \notin X_{l} \text { or vice versa } \\ 1 & \text { otherwise }\end{cases}
$$

(a pair $\{i, j\}$ such that $i \in X_{l}$ and $j \notin X_{l}$ or vice versa is said to "cross" $X_{l}$ ).

- The measure

$$
\int d \mu_{\tau}\left(\mathbf{t}_{n-1}, \mathbf{X}_{n-1}\right)[\cdots] \doteq \int_{0}^{1} d t_{1} \ldots \int_{0}^{1} d t_{n-1} \sum_{\substack{\mathbf{X}_{n-1} \\ \text { comp. } \tau}} t_{1}^{b_{1}-1} \ldots t_{n-1}^{b_{n-1}-1}[\cdots]
$$

has total mass equal to one (i.e. it is a probability measure). In (3.3) " $\mathbf{X}_{n-1}$ comp. $\tau$ " means that for all $i=1,2, \ldots, n-1, X_{i}$ contains exactly $i-1$ edges of $\tau$ and $b_{i}$ is the numebr of edges in $\tau$ which "cross" $X_{i}$

We want to use (3.1) to bond $\left|\phi^{T}\left(\gamma_{1}, \ldots, \gamma_{n}\right)\right|$. This formula is useful when the pair potential is not purely repulsive. However, due to the restriction $V_{i j}$ finite (otherwise the r.h.s. of (3.1) is not well defined), one in general can apply (3.1) only if the pair potential is finite and absolutely integrable, see [4], which is a quite restrictive condition. In particular this rules out a pair potential with hard core at short distances which is precisely one of the situations we would like to treat.

We show here that it is possible to give meaning to r.h.s. of (3.1) even when some among the $V_{i j}$ 's take the value $\infty$ (the 1.h.s. of (3.1) makes sense even in this case). We define a cut-offed pair potential

$$
V_{H}\left(\gamma_{i}, \gamma_{j}\right)= \begin{cases}H & \text { if } \gamma_{i} \nsim \gamma_{j} \\ V\left(\gamma_{i}, \gamma_{j}\right) & \text { otherwise }\end{cases}
$$

Note that, from stability condition (2.3), for any fixed $n \in \mathbb{N}$ and $\left(\gamma_{1}, \ldots, \gamma_{n}\right) \in \mathcal{P}^{n}$, there is $H_{0}$ (which depends on $n$ and $\left.\left(\gamma_{1}, \ldots, \gamma_{n}\right) \in \mathcal{P}^{n}\right)$ such that, for all $H \geq H_{0}$ and for all $X \subset\{1,2, \ldots, n\}$,

$$
\sum_{\{i, j\} \subset X} V_{H}\left(\gamma_{i}, \gamma_{j}\right) \geq-\sum_{i \in X} B\left(\gamma_{i}\right)
$$

Indeed, if $X \subset\{1,2, \ldots, n\}$ is such that $\left\{\gamma_{i}\right\}_{i \in X}$ does not contain incompatible pairs, then, by definition (3.4) and inequality (2.3), it follows

$$
\sum_{\{i, j\} \subset X} V_{H}\left(\gamma_{i}, \gamma_{j}\right)=\sum_{\{i, j\} \subset X} V\left(\gamma_{i}, \gamma_{j}\right) \geq \sum_{i \in X} B\left(\gamma_{i}\right)
$$

If $X$ is such that $\left\{\gamma_{i}\right\}_{i \in X}$ does contain incompatible pairs, then there is at least an edge $\{k, s\}$ such that $V^{H}\left(\gamma_{i}, \gamma_{j}\right)=H$ so taking

$$
H_{0}^{X}=-\sum_{\substack{\{i, j\} \in X \\ V\left(\gamma_{i}, \gamma_{j}\right) \leq 0,\{i, j\} \neq\{k, s\}}} V\left(\gamma_{i}, \gamma_{j}\right)
$$

we have, whenever $H \geq H_{0}^{X}$

$$
\sum_{\{i, j\} \subset X} V_{H}\left(\gamma_{i}, \gamma_{j}\right) \geq 0 \geq-\sum_{i \in X}^{n} B\left(\gamma_{i}\right)
$$


So, taking $H_{0}=\max _{X \subset\{1,2, \ldots, n\}} H_{0}^{X}$ the inequalities (3.5) are satisfied for all $X \subset\{1,2, \ldots, n\}$.

Now, for any fixed $\left(\gamma_{1}, \ldots, \gamma_{n}\right) \in \mathcal{P}^{n}$

$$
\phi^{T}\left(\gamma_{1}, \ldots, \gamma_{n}\right)=\lim _{H \rightarrow \infty} \sum_{g \in G_{n}} \prod_{\{i, j\} \in E_{g}}\left(e^{-V_{H}\left(\gamma_{i}, \gamma_{j}\right)}-1\right)
$$

We can now use (3.1) for the finite potential $V_{H}$ and we get

$$
\begin{gathered}
\sum_{g \in G_{n}} \prod_{\{i, j\} \in E_{g}}\left(e^{-V_{H}\left(\gamma_{i}, \gamma_{j}\right)}-1\right)= \\
=\lim _{H \rightarrow \infty} \sum_{\tau \in T_{n}} \prod_{\{i, j\} \in E_{\tau}}\left(-V_{H}\left(\gamma_{i}, \gamma_{j}\right)\right) \int d \mu_{\tau}\left(\mathbf{t}_{n-1}, \mathbf{X}_{n-1}\right) e^{-K_{H}\left(\mathbf{X}_{n-1}, \mathbf{t}_{n-1}\right)}
\end{gathered}
$$

where

$$
K_{H}\left(\mathbf{X}_{n-1}, \mathbf{t}_{n-1}\right)=\sum_{1 \leq i<j \leq n} t_{1}(\{i, j\}) \ldots t_{n-1}(\{i, j\}) V_{H}\left(\gamma_{i}, \gamma_{j}\right)
$$

Now, for fixed $\tau=\left(\mathrm{I}_{n}, E_{\tau}\right) \in T_{n}$ and $\left(\gamma_{1}, \ldots, \gamma_{n}\right) \in \mathcal{P}^{n}$, let us consider the factor

$$
w_{H}^{\tau}\left(\gamma_{1}, \ldots, \gamma_{n}\right)=\prod_{\{i, j\} \in E_{\tau}}\left|V_{H, J}\left(\gamma_{i}, \gamma_{j}\right)\right| \int d \mu_{\tau}\left(\mathbf{t}_{n-1}, \mathbf{X}_{n-1}\right) e^{-K_{H}\left(\mathbf{X}_{n-1}, \mathbf{t}_{n-1}\right)}
$$

The edges $\{i, j\} \subset \mathrm{I}_{n}$ are naturally partitioned into two disjoint sets $\mathrm{E}_{n}^{H}$ and $\mathrm{E}_{n} \backslash \mathrm{E}_{n}^{H}$ where $\mathrm{E}_{n}^{H}=$ $\left.\left\{\{i, j\} \subset I_{n}: \gamma_{i} \not \gamma_{j}\right)\right\}$. Thus also the edges of the tree $\tau$ are partitioned into two disjoint sets $E_{\tau}^{H}$ and $E_{\tau} \backslash E_{\tau}^{H}$ where $E_{\tau}^{H}=E_{\tau} \cap \mathrm{E}_{n}^{H}$. So we have

$$
w_{H}^{\tau}\left(\gamma_{1}, \ldots, \gamma_{n}\right)=\prod_{\{i, j\} \in E_{\tau} \backslash E_{\tau}^{H}}\left|V_{H}\left(\gamma_{i}, \gamma_{j}\right)\right| \prod_{\{i, j\} \in E_{\tau}^{H}}\left|V_{H}\left(\gamma_{i}, \gamma_{j}\right)\right| \int d \mu_{\tau}\left(\mathbf{t}_{n-1}, \mathbf{X}_{n-1}\right) e^{-K_{H}\left(\mathbf{X}_{n-1}, \mathbf{t}_{n-1}\right)}
$$

Now, recalling the definition (3.6), we can write

$$
K_{H}\left(\mathbf{X}_{n-1}, \mathbf{t}_{n-1}\right)=K_{U_{(1-\varepsilon) H}}\left(\mathbf{X}_{n-1}, \mathbf{t}_{n-1}\right)+K_{V_{\varepsilon H}}\left(\mathbf{X}_{n-1}, \mathbf{t}_{n-1}\right)
$$

where

$$
K_{U_{(1-\varepsilon) H}}\left(\mathbf{X}_{n-1}, \mathbf{t}_{n-1}\right)=\sum_{1 \leq i<j \leq n} t_{1}(\{i, j\}) \ldots t_{n-1}(\{i, j\}) U_{(1-\varepsilon) H}\left(\gamma_{i}, \gamma_{j}\right)
$$

and

$$
K_{V_{\varepsilon H}}\left(\mathbf{X}_{n-1}, \mathbf{t}_{n-1}\right)=\sum_{1 \leq i<j \leq n} t_{1}(\{i, j\}) \ldots t_{n-1}(\{i, j\}) V_{\varepsilon H}\left(\gamma_{i}, \gamma_{j}\right)
$$

where $\varepsilon>0$ and

$$
U_{(1-\varepsilon) H}\left(\gamma_{i}, \gamma_{j}\right)= \begin{cases}(1-\varepsilon) H & \text { if } \gamma_{i} \nsim \gamma_{j} \\ 0 & \text { otherwise }\end{cases}
$$


and

$$
V_{\varepsilon H}\left(\gamma_{i}, \gamma_{j}\right)= \begin{cases}\varepsilon H & \text { if } \gamma_{i} \nsim \gamma_{j} \\ V\left(\gamma_{i}, \gamma_{j}\right) & \text { otherwise }\end{cases}
$$

The potential $t_{1}(\{i, j\}) \ldots t_{n-1}(\{i, j\}) V_{\varepsilon H}\left(\gamma_{i}, \gamma_{j}\right)$ satisfies, for $H$ larger that $\varepsilon^{-1} H_{0}$

$$
\sum_{\{i, j\} \subset X} V_{\varepsilon H}\left(\gamma_{i}, \gamma_{j}\right) \geq-\sum_{i \in X} B\left(\gamma_{i}\right)
$$

for all $X \subset\{1,2, \ldots, n\}$. This fact implies (see e.g. [4], [16], 17]) that

$$
K_{V_{\varepsilon H}}\left(\mathbf{X}_{n-1}, \mathbf{t}_{n-1}\right) \geq-\sum_{i=1}^{n} B\left(\gamma_{i}\right)
$$

The potential $K_{U_{(1-\varepsilon) H}}\left(\mathbf{X}_{n-1}, \mathbf{t}_{n-1}\right)$ is non negative and can be bounded, for $\eta>0$, as follows

$$
\begin{gathered}
K_{U_{(1-\varepsilon) H}}\left(\mathbf{X}_{n-1}, \mathbf{t}_{n-1}\right) \geq \sum_{\{i, j\} \subset E_{\tau}^{H}} t_{1}(\{i, j\}) \ldots t_{n-1}(\{i, j\}) U_{(1-\varepsilon) H}\left(\gamma_{i}, \gamma_{j}\right)= \\
=\sum_{\{i, j\} \subset E_{\tau}^{H}} t_{1}(\{i, j\}) \ldots t_{n-1}(\{i, j\})(1-\varepsilon) H+\eta \sum_{\{i, j\} \subset E_{\tau} \backslash E_{\tau}^{H}} t_{1}(\{i, j\}) \ldots t_{n-1}(\{i, j\})- \\
-\eta \sum_{\{i, j\} \subset E_{\tau} \backslash E_{\tau}^{H}} t_{1}(\{i, j\}) \ldots t_{n-1}(\{i, j\}) \geq \\
\geq \sum_{\{i, j\} \subset E_{\tau}^{H}} t_{1}(\{i, j\}) \ldots t_{n-1}(\{i, j\})(1-\varepsilon) H+\sum_{\{i, j\} \subset E_{\tau} \backslash E_{\tau}^{H}} t_{1}(\{i, j\}) \ldots t_{n-1}(\{i, j\}) \eta-\left|E_{\tau} \backslash E_{\tau}^{H}\right| \eta
\end{gathered}
$$

So we get

$$
K_{U_{(1-\varepsilon) H}}\left(\mathbf{X}_{n-1}, \mathbf{t}_{n-1}\right) \geq \sum_{1 \leq i<j \leq n} t_{1}(\{i, j\}) \ldots t_{n-1}(\{i, j\}) V_{i j}^{\tau}-\left|E_{\tau} \backslash E_{\tau}^{H}\right| \eta
$$

where $V_{i j}^{\tau}$ is the positive $(H, \eta, \varepsilon$ dependent) pair potential given by

$$
V_{i j}^{\tau}= \begin{cases}(1-\varepsilon) H & \text { if }\{i, j\} \in E_{\tau}^{H} \\ \eta & \text { if }\{i, j\} \in E_{\tau} \backslash E_{\tau}^{H} \\ 0 & \text { otherwise }\end{cases}
$$

Hence, plugging (3.8) and (3.9) into (3.7) we obtain that $w_{H}^{\tau}\left(\gamma_{1}, \ldots, \gamma_{n}\right)$ can be bounded by

$$
\begin{gathered}
w_{H}^{\tau}\left(\gamma_{1}, \ldots, \gamma_{n}\right) \leq e^{+\sum_{i=1}^{n} B\left(\gamma_{i}\right)+\eta\left|E_{\tau} \backslash E_{\tau}^{H}\right|}\left[\prod_{\{i, j\} \in E_{\tau} \backslash E_{\tau}^{H}}\left|V\left(\gamma_{i}, \gamma_{j}\right)\right|\right] \times\left[\frac{1}{\eta}\right]^{\left|E_{\tau} \backslash E_{\tau}^{H}\right|} \times \\
\times\left[\frac{1}{1-\varepsilon}\right]^{\left|E_{\tau}^{H}\right|} \prod_{\{i, j\} \in E_{\tau}} V_{i j}^{\tau} \int d \mu_{\tau}\left(\mathbf{t}_{n-1}, \mathbf{X}_{n-1}\right) e^{-\sum_{1 \leq i<j \leq n} t_{1}(\{i, j\}) \ldots t_{n-1}(\{i, j\}) V_{i j}^{\tau}}
\end{gathered}
$$


Applying now the tree graph identity (3.1) to the pair potential $V_{i j}^{\tau}$ one conclude immediately (see e.g. 4]) that, for all $H \in[0,+\infty)$

$$
\begin{aligned}
& \prod_{\{i, j\} \in E_{\tau}} V_{i j}^{\tau} \int d \mu_{\tau}\left(\mathbf{t}_{n-1}, \mathbf{X}_{n-1}\right) e^{-\sum_{1 \leq i<j \leq n} t_{1}(\{i, j\}) \ldots t_{n-1}(\{i, j\}) V_{i j}^{\tau}}=\prod_{\{i, j\} \in E_{\tau}}\left|e^{-V_{i j}^{\tau}}-1\right|= \\
& =\left|e^{-(1-\varepsilon) H}-1\right|^{\left|E_{\tau}^{H}\right|}\left|e^{-\eta}-1\right|^{\left|E_{\tau} \backslash E_{\tau}^{H}\right|}=\left|e^{-\eta}-1\right|^{\left|E_{\tau} \backslash E_{\tau}^{H}\right|} \prod_{\{i, j\} \in E_{\tau}^{H}}\left|e^{-U_{(1-\varepsilon) H}\left(\gamma_{i}, \gamma_{j}\right)}-1\right|
\end{aligned}
$$

Hence, considering that $e^{\eta\left|E_{\tau} \backslash E_{\tau}^{H}\right|}\left|e^{-\eta}-1\right|^{\left|E_{\tau} \backslash E_{\tau}^{H}\right|}=\left(e^{\eta}-1\right)^{\left|E_{\tau} \backslash E_{\tau}^{H}\right|}$ we get

$$
w_{H}^{\tau}\left(\gamma_{1}, \ldots, \gamma_{n}\right) \leq e^{+\sum_{i=1}^{n} B\left(\gamma_{i}\right)} \prod_{\{i, j\} \in E_{\tau}^{H}}\left[\frac{1}{1-\varepsilon}\right]\left|e^{-U_{(1-\varepsilon) H}\left(\gamma_{i}, \gamma_{j}\right)}-1\right| \prod_{\{i, j\} \in E_{\tau} \backslash E_{\tau}^{H}}\left|\frac{\left(e^{\eta}-1\right)}{\eta} V\left(\gamma_{i}, \gamma_{j}\right)\right|
$$

and due to the arbitrarity of $\eta$ and $\varepsilon$ which can be taken as small as we please, and using also that $U_{(1-\varepsilon) H}\left(\gamma_{i}, \gamma_{j}\right)<V\left(\gamma_{i}, \gamma_{j}\right)$ for any $\gamma_{i} \not \gamma_{j}$ and any finite $H$, we obtain, for any $H \geq H_{0}$

$$
w_{H}^{\tau}\left(\gamma_{1}, \ldots, \gamma_{n}\right) \leq e^{+\sum_{i=1}^{n} B\left(\gamma_{i}\right)} \prod_{\{i, j\} \in E_{\tau}^{H}}\left|e^{-V\left(\gamma_{i}, \gamma_{j}\right)}-1\right| \prod_{\{i, j\} \in E_{\tau} \backslash E_{\tau}^{H}}\left|V\left(\gamma_{i}, \gamma_{j}\right)\right|
$$

which is a bound independent of $H$. So

$$
w^{\tau}\left(\gamma_{1}, \ldots, \gamma_{n}\right)=\lim _{H \rightarrow \infty} w_{H}^{\tau}\left(\gamma_{1}, \ldots, \gamma_{n}\right) \leq e^{+\sum_{i=1}^{n} B\left(\gamma_{i}\right)} \prod_{\{i, j\} \in E_{\tau}^{H}}\left|e^{-V\left(\gamma_{i}, \gamma_{j}\right)}-1\right| \prod_{\{i, j\} \in E_{\tau} \backslash E_{\tau}^{H}}\left|V\left(\gamma_{i}, \gamma_{j}\right)\right|
$$

In conclusion we have that

$$
\left|\phi^{T}\left(\gamma_{1}, \ldots, \gamma_{n}\right)\right| \leq e^{+\sum_{i=1}^{n} B\left(\gamma_{i}\right)} \sum_{\tau \in T_{n}} \prod_{\{i, j\} \in E_{\tau}} F\left(\gamma_{i}, \gamma_{j}\right)
$$

where

$$
F\left(\gamma_{i}, \gamma_{j}\right)= \begin{cases}\left|e^{-V\left(\gamma_{i}, \gamma_{j}\right)}-1\right| & \text { if } \gamma_{i} \nsim \gamma_{j} \\ \left|V\left(\gamma_{i}, \gamma_{j}\right)\right| & \text { otherwise }\end{cases}
$$

and hence also, for $n \geq 1$

$$
\left|\phi^{T}\left(\gamma_{0}, \gamma_{1}, \ldots, \gamma_{n}\right)\right| \leq e^{+\sum_{i=0}^{n} B\left(\gamma_{i}\right)} \sum_{\tau \in T_{n}^{0}} \prod_{\{i, j\} \in E_{\tau}} F\left(\gamma_{i}, \gamma_{j}\right)
$$

where $T_{n}^{0}$ is the set of all trees with vertex set $\mathrm{I}_{n}^{0} \doteq\{0,1,2, \ldots, n\}$. Inserting (3.12) in (2.7) we get

$$
\Pi_{\mathcal{P}}^{\gamma_{0}}(\rho) \leq 1+\sum_{n=1}^{\infty} \frac{1}{n !} \sum_{\left(\gamma_{1}, \gamma_{2}, \ldots, \gamma_{n}\right) \in \mathcal{P}^{n}} e^{+\sum_{i=0}^{n} B\left(\gamma_{i}\right)} \sum_{\tau \in T_{n}^{0}} \prod_{\{i, j\} \in E_{\tau}} F\left(\gamma_{i}, \gamma_{j}\right) \rho_{\gamma_{1}} \ldots \rho_{\gamma_{n}} \leq
$$




$$
\leq e^{B\left(\gamma_{0}\right)}\left[1+\sum_{n=1}^{\infty} \frac{1}{n !} \sum_{\left(\gamma_{1}, \ldots, \gamma_{n}\right) \in \mathcal{P}^{n}} \sum_{\tau \in T_{n}^{0}} \prod_{\{i, j\} \in E_{\tau}} F\left(\gamma_{i}, \gamma_{j}\right) \rho_{\gamma_{1}} e^{B\left(\gamma_{1}\right)} \ldots \rho_{\gamma_{n}} e^{B\left(\gamma_{n}\right)}\right]=
$$

If we pose

$$
\tilde{\rho}_{\gamma}=\rho_{\gamma} e^{B(\gamma)}
$$

and

$$
|\tilde{\Pi}|_{\mathcal{P}}^{\gamma_{0}}(\tilde{\rho})=1+\sum_{n=1}^{\infty} \frac{1}{n !} \sum_{\tau \in T_{n}^{0}} \sum_{\left(\gamma_{1}, \ldots, \gamma_{n}\right) \in \mathcal{P}^{n}} \prod_{\{i, j\} \in E_{\tau}} F\left(\gamma_{i}, \gamma_{j}\right) \tilde{\rho}_{\gamma_{1}} \ldots \tilde{\rho}_{\gamma_{n}}
$$

We get

$$
|\Pi|_{\mathcal{P}}^{\gamma_{0}}(\rho) \leq e^{B\left(\gamma_{0}\right)}|\tilde{\Pi}|_{\mathcal{P}}^{\gamma_{0}}(\tilde{\rho})
$$

So the convergence of $|\tilde{\Pi}|_{\mathcal{P}}^{\gamma_{0}}(\tilde{\rho})$ implies that of $|\Pi|_{\mathcal{P}}^{\gamma_{0}}(\rho)$.

\subsection{Planar rooted trees and convergence}

We think the trees with vertex set $\mathrm{I}_{n}^{0}=\{0,1,2, \ldots, n\}$ (i.e the elements of $T_{n}^{0}$ ) as rooted in 0 . We define a map $m: \tau \mapsto m(\tau)$ which associate to each labelled tree $\tau \in T_{n}^{0}$ a unique drawing $t=m(\tau)$ in the plane, called the planar rooted tree associated to $\tau$, as follows.

Given $\tau$ in $T_{n}^{0}$, place the vertex 0 (the root) at the leftmost position of the drawing. From 0 there emerge $s_{0}$ branches ending at the first-generation vertices $i_{1}, \ldots, i_{s_{0}}$. Drawn these vertices along a vertical line at the right of the root in such way that the higher has the low label and labels increase as we go down along the vertical line (ordering increasing label vertices "from high to low"). Then iterate this procedure for the descendants of each first generation vertex (i.e. the second generation vertices) $i_{1}, \ldots i_{s_{0}}$ and so on... (see figure 1).

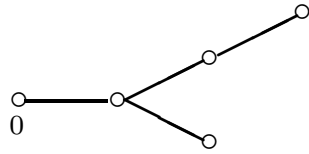

(a)

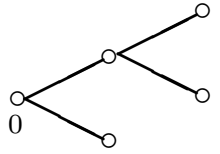

(b)

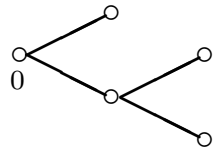

(c)

Figure 1: the planar rooted trees associated to the trees (a) with edge set $\{0,3\},\{1,3\},\{2,3\},\{1,4\},(b)$ with edge set $\{0,2\},\{0,3\},\{1,2\},\{2,4\}$ and (c) with edge set $\{0,2\},\{0,4\},\{4,3\},\{1,4\}$. Observe that (b) and (c) are different planar rooted tree

There is a natural partial order $\prec$ among the vertices in a rooted tree. For $u, v \in t$, we say that $u$ precedes $v$ and write $u \prec v$ (or $v \succ u$ ) if the (unique) path from the root to $v$ contains $u$. If $\{v, u\}$ is an edge of $t$ rooted tree, then either $v \prec u$ or $u \prec v$. If $u \prec v$. $u$ is called the predecessor and $v$ is called the descendant. The root has no predecessor and it is the extremum respect to the partial order relation $\prec$ in $t$. For each vertex $v$ of $t$, we will denote by $s_{v}$ the branching factor of $v$ and we denote by $v^{1} \ldots, v^{s_{v}}$ the $s_{v}$ descendants of $v,\left(v^{1}\right.$ being the higher and $v^{s_{v}}$ being the lower in the drawing). 
Clearly the map $\tau \mapsto m(\tau)=t$ is many-to-one and the cardinality of the preimage of a planar rooted tree $t$ (=number of ways of labelling the $n$ non-root vertices of the tree with $n$ distinct labels consistently with the rule "from high to low") is given by

$$
\left|\left\{\tau \in T_{n}^{0}: m(\tau)=t\right\}\right|=\frac{n !}{\prod_{v \succeq 0} s_{v_{i}} !}
$$

We denote by $\mathbb{T}_{n}^{0}=$ the set of all planar rooted trees with $n$ vertices and by $\mathbb{T}^{0, k}$ the set of planar rooted trees with maximal generation number $k$; let also $\mathbb{T}^{0}=\cup_{n \geq 0} \mathbb{T}_{n}^{0}=\cup_{k \geq 0} \mathbb{T}^{0, k}$ be the set of all planar rooted trees.

Let now $\mu: \mathcal{P} \rightarrow[0, \infty)^{\mathcal{P}}: \gamma \mapsto \mu_{\gamma}$ be a positive valued function defined in $\mathcal{P}$ and let, for each $\gamma \in \mathcal{P}, R_{\gamma} \in[0, \infty)^{\mathcal{P}}$ be defined by the equations

$$
\mu_{\gamma}=R_{\gamma} \varphi_{\gamma}(\mu), \quad \gamma \in \mathcal{P}
$$

with

$$
\varphi_{\gamma}(\mu)=1+\sum_{n \geq 1} \sum_{\left(\gamma_{1}, \ldots, \gamma_{n}\right) \in \mathcal{P}^{n}} b_{n}\left(\gamma ; \gamma_{1}, \ldots, \gamma_{n}\right) \mu_{\gamma_{1}} \ldots \mu_{\gamma_{n}}
$$

for certain functions $b_{n}: \mathcal{P}^{n+1} \rightarrow[0, \infty)$. Denoting $R_{\gamma} \varphi_{\gamma}(\mu)=T_{\gamma_{0}}(\mu)$ the equation $(3.17)$ can be visualized in the diagrammatic form

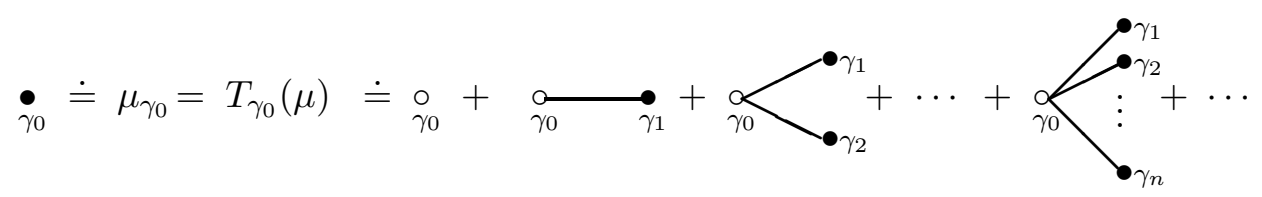

The sum is over all single-generation rooted trees. In each tree, vertices with open circles with subscript $\gamma$ represents a factor $R_{\gamma}$, vertices with bullets with subscript $\gamma$ a factor $\mu_{\gamma}$ and vertices other than the root must be summed over all possible polymers $\gamma$. At each vertex with $n$ descendants, a "vertex function" $b_{n}$ acts, having as arguments the $n+1$-tuple formed by the polymer at the vertex and the $n$ polymers associated to the $n$ descendants of that vertex. With this representation, the iteration $T^{2}(\mu)=T(T(\mu))$ corresponds to replacing each of the bullets by each one of the diagrams of the expansion for $T$. This leads to planar rooted trees of up to two generations, with open circles at first-generation vertices and bullets at second-generation ones. In particular, all single-generation trees have only open circles. Notice that the two drawings of Figure 1 appear in two different terms of the expansion, and hence should be counted as different diagrams. More generally, the $k$-th iteration of $T$ involves all possible planar rooted trees up to $k$ generations. In each term of the expansion, vertices of generation $k$ are occupied by bullets and all the others by open circles. A straightforward inductive argument shows that

$$
T_{\gamma_{0}}^{k}(\mu)=R_{\gamma_{0}}\left[\sum_{\ell=0}^{k-1} \Phi_{\gamma_{0}}^{(\ell)}(R)+\Phi_{\gamma_{0}}^{(k)}(R, \mu)\right]
$$

where we have denoted $R=\left\{R_{\gamma}\right\}_{\gamma \in \mathcal{P}}$ and

$$
\Phi_{\gamma_{0}}^{(\ell)}(R)=\sum_{t \in \mathbb{T}^{0}, \ell} \prod_{v \succeq 0}\left\{\sum_{\left(\gamma_{v}, \ldots, \gamma_{v^{s_{v}}}\right) \in \mathcal{P}^{s_{v}}} b_{s_{v}}\left(\gamma_{v} ; \gamma_{v^{1}}, \ldots, \gamma_{v^{s_{v}}}\right) R_{\gamma_{v^{1}}} \ldots R_{\gamma_{v} s_{v}}\right\}
$$


Here the product $\prod_{v \succeq 0}$ over the vertices of $t$ must be done respecting the partial order of the set of vertices in $t$, i.e. if $v \succ u$ the $v$ must be at the right of $u$ in the product. The factor $\Phi_{\gamma_{0}}^{(k)}(R, \mu)$ has a similar expression but with the activities of the vertex of the $k$-th generation weighted by $\mu$. Here we agree that $b_{0}\left(\gamma_{v}\right) \equiv 1$ and $\prod_{\emptyset} \equiv 1$. We are interested in the $\ell \rightarrow \infty$ limit of (3.20).

Proposition 2 Let $\mu: \mathcal{P} \rightarrow[0, \infty)^{\mathcal{P}}: \gamma \mapsto \mu_{\gamma}$ be a positive valued function and let, for each $\gamma \in \mathcal{P}$, $R_{\gamma} \in[0, \infty)^{\mathcal{P}}$ be defined by the equations (3.17). Let, $\forall \gamma \in \mathcal{P}, \tilde{\rho}_{\gamma} \in[0, \infty)$ such that $\tilde{\rho}_{\gamma} \leq R_{\gamma}$. Then the series

$$
\Phi_{\gamma_{0}}(\tilde{\rho}):=\sum_{t \in \mathbb{T}^{0}} \prod_{v \succeq 0}\left\{\sum_{\left(\gamma_{v}, \ldots, \gamma_{v^{s_{v}}}\right) \in \mathcal{P}^{s_{v}}} b_{s_{v}}\left(\gamma_{v} ; \gamma_{v^{1}}, \ldots, \gamma_{v^{s_{v}}}\right) \tilde{\rho}_{\gamma_{v^{1}}} \ldots \tilde{\rho}_{\gamma_{v} s_{v}}\right\}
$$

is finite for each $\gamma_{0} \in \mathcal{P}$. Furthermore

$$
\tilde{\rho}_{\gamma_{0}} \Phi_{\gamma_{0}}(\tilde{\rho}) \leq \mu_{\gamma_{0}}
$$

for each $\gamma_{0} \in \mathcal{P}$.

Proof. By definition $\Phi_{\gamma_{0}}(\tilde{\rho})=\sum_{\ell=0}^{\infty} \Phi_{\gamma_{0}}^{(\ell)}(\tilde{\rho})$. By (3.19), the fact that $T_{\gamma_{0}}^{k}(\mu)=\mu_{\gamma_{0}}$ for all $k \in \mathbb{N}$, and the assumption $\tilde{\rho}_{\gamma} \leq R_{\gamma}$ for all $\gamma \in \mathcal{P}$, we obtain that

$$
\tilde{\rho}_{\gamma_{0}} \sum_{\ell=0}^{n} \Phi_{\gamma_{0}}^{(\ell)}(\tilde{\rho}) \leq R_{\gamma_{0}} \sum_{\ell=0}^{n} \Phi_{\gamma_{0}}^{(\ell)}(R) \leq \mu_{\gamma_{0}}
$$

for all $n$. Thus, since the sequence of partial sums of the series $\rho_{\gamma_{0}} \Phi_{\gamma_{0}}(\tilde{\rho})$ is monotonic increasing and bounded by $\mu_{\gamma_{0}}, \tilde{\rho}_{\gamma_{0}} \Phi_{\gamma_{0}}(\tilde{\rho})$ converges, and $\tilde{\rho}_{\gamma_{0}} \Phi_{\gamma_{0}}(\tilde{\rho}) \leq \mu_{\gamma_{0}}$.

\subsection{End of the proof of theorem 1}

We first reorganize the sum over labelled trees appearing in formula (3.14) in terms of the called planar rooted trees previously introduced. Namely, recalling that $\mathbb{T}_{n}^{0}$ is the set of all planar rooted trees with fixed root 0 and $n$ vertices (different from the root), we can rewrite the r.h.s. of (3.14) as

$$
|\tilde{\Pi}|_{\mathcal{P}}^{\gamma_{0}}(\tilde{\rho})=1+\sum_{n=1}^{\infty} \frac{1}{n !} \sum_{t \in \mathbb{T}_{n}^{0}} \sum_{\substack{\tau \in T_{n}^{0} \\ m(\tau)=t}} \sum_{\left(\gamma_{1}, \ldots, \gamma_{n}\right) \subset \mathcal{P}^{n}} \prod_{\{i, j\} \in E_{\tau}} F\left(\gamma_{i}, \gamma_{j}\right) \tilde{\rho}_{\gamma_{1}} \ldots \tilde{\rho}_{\gamma_{n}}
$$

Observe that the factor

$$
\sum_{\left(\gamma_{1}, \ldots, \gamma_{n}\right) \subset \mathcal{P}^{n}} \prod_{\{i, j\} \in E_{\tau}} F\left(\gamma_{i}, \gamma_{j}\right) \tilde{\rho}_{\gamma_{1}} \ldots \tilde{\rho}_{\gamma_{n}}
$$

depends only on the planar rooted tree $t=m(\tau)$ associated to $\tau$ (labels of $\tau$ are dummy indices in the sum), i.e.

$$
\sum_{\left(\gamma_{1}, \ldots, \gamma_{n}\right) \subset \mathcal{P}^{n}} \prod_{\{i, j\} \in E_{\tau}} F\left(\gamma_{i}, \gamma_{j}\right) \tilde{\rho}_{\gamma_{1}} \ldots \tilde{\rho}_{\gamma_{n}}=\prod_{v \succeq v_{0}}\left\{\prod_{i=1}^{s_{v}} \sum_{\gamma_{v^{i}} \in \mathcal{P}} F\left(\gamma_{v}, \gamma_{v^{i}}\right) \tilde{\rho}_{\gamma_{v^{i}}}\right\}
$$


with the convention that $\prod_{i=1}^{s_{v}} \sum_{\gamma_{v^{i}} \in \mathcal{P}} F\left(\gamma_{v}, \gamma_{v^{i}}\right) \tilde{\rho}_{\gamma_{v^{i}}}=1$ when $s_{v}=0$.

So in conclusion, inserting (3.24) into (3.23) and using also (3.16), we obtain

$$
|\tilde{\Pi}|_{\mathcal{P}}^{\gamma_{0}}(\tilde{\rho})=\sum_{t \in \mathbb{T}^{0}} \prod_{v \succeq v_{0}} \frac{1}{s_{v} !}\left\{\prod_{i=1}^{s_{v}} \sum_{\gamma_{v^{i}} \in \mathcal{P}} F\left(\gamma_{v}, \gamma_{v^{i}}\right) \tilde{\rho}_{\gamma_{v^{i}}}\right\}
$$

Comparing (3.25) with $(3.21)$ we immediately see that $|\tilde{\Pi}|_{\mathcal{P}}^{\gamma_{0}}(\tilde{\rho})=\Phi_{\gamma_{0}}(\tilde{\rho})$ provided

$$
b_{n}\left(\gamma ; \gamma_{1}, \ldots, \gamma_{n}\right)=\frac{1}{n !} \prod_{i=1}^{n} F\left(\gamma, \gamma_{i}\right)
$$

so that

$$
\varphi_{\gamma}(\mu)=1+\sum_{n \geq 1} \frac{1}{n !} \sum_{\left(\gamma_{1}, \ldots, \gamma_{n}\right) \in \mathcal{P}^{n}} \prod_{i=1}^{n} F\left(\gamma, \gamma_{i}\right) \mu_{\gamma_{i}}=e^{\sum_{\tilde{\gamma} \in \mathcal{P}} F(\gamma, \tilde{\gamma}) \mu_{\gamma}}
$$

Hence proposition 2 yields the criterion (2.11) for the convergence of the series $|\tilde{\Pi}|_{\mathcal{P}}^{\gamma_{0}}(\tilde{\rho})$ defined in (2.7). As a matter of fact, by proposition 2, with the identification (3.26), we have immediately that the series $|\tilde{\Pi}|_{\mathcal{P}}^{\gamma_{0}}(\tilde{\rho})$ defined in (3.14) is finite for each $\gamma_{0} \in \mathcal{P}$ and $\tilde{\rho}_{\gamma_{0}}|\tilde{\Pi}|_{\mathcal{P}}^{\gamma_{0}}(\tilde{\rho}) \leq \mu_{\gamma_{0}}$ for each $\gamma_{0} \in \mathcal{P}$. Now recalling (3.15) and (3.13) we obtain $\rho_{\gamma_{0}} \Pi_{\gamma_{0}}(\rho) \leq \mu_{\gamma_{0}}$.

\section{Example. BEG model with infinite range interactions in the low temperature disordered phase}

As an example, we consider the Blume-Emery-Griffiths (BEG) model [2] with infinite range interactions in the low temperature disordered phase. The model is defined on the cubic unit lattice in $d$-dimensions $\mathbb{Z}^{d}$ by supposing that in each vertex $x \in \mathbb{Z}^{d}$ there is a spin variable $\sigma_{x}$ taking values in the set $\{0,-1,+1\}$. These spins interact via the (formal) Hamiltonian

$$
H=-\sum_{\{x, y\} \subset \mathbb{Z}^{d}}\left[J_{x y} \sigma_{x} \sigma_{y}+K_{x y} \sigma_{x}^{2} \sigma_{y}^{2}\right]+D \sum_{x \in \mathbb{Z}^{d}} \sigma_{x}^{2}
$$

where $J_{x y} \geq 0$ and $K_{x y} \in \mathbb{R}$ are summable interactions and we put

$$
J=\frac{1}{2} \sup _{x \in \mathbb{Z}^{d}} \sum_{\substack{y \in \mathbb{Z}^{d} \\ y \neq x}}\left(J_{x y}+\left|K_{x y}\right|\right)
$$

In the region of parameters

$$
D>J
$$

the ground state is $\sigma=0$. This region is called the disordered phase. If $J_{x y}$ and $K_{x y}$ are nearest neighbor interactions (or finite range), the low temperature disordered phase can be studied using the standard Pirogov-Sinai theory.

We will make here different assumptions on the interactions $J_{x y}$ and $K_{x y}$. Namely, we suppose that there exist positive constants $c, J_{1}, \lambda$ and $\lambda^{\prime}$ (with $0<\lambda<\lambda^{\prime}$ ) such that 


$$
J_{x y}+\left|K_{x y}\right| \leq \frac{2 J_{1}}{|x-y|^{d+\lambda}} \quad \forall\{x, y\} \in \mathbb{Z}^{d}
$$

and

$$
J_{x y} \geq \frac{c}{|x-y|^{d+\lambda^{\prime}}} \quad \text { or } \quad\left|K_{x y}\right| \geq \frac{c}{|x-y|^{d+\lambda^{\prime}}}
$$

where $|x-y|$ is the usual nearest neighbor path distance, i.e., $|x-y|$ is the length of the shortest path of nearest neighbors connecting $x$ to $y$. Due to the assumption (4.5) the low temperature phase of the BEG model described by the Hamiltonian (4.1), cannot be studied using the standard low temperature Pirogov-Sinai, which explicitly requires finite range interactions. If we further assume that the polynomial decay is slow, e.g. by supposing

$$
\lambda^{\prime}<2 d+1
$$

then this model is not even included in the class of models whose low temperature phase can be studied via the extension of the Pirogov-Sinai theory to infinite range interactions given in [13.

We'll show in this section that the partition function of the spin model described by Hamiltonian (4.1) can rewritten as the partition function of a polymer system of the type considered in the previous sections. Then, using theorem 1, we will prove that, in the disordered phase (4.3) and with the assumptions (4.4), (4.5), (4.6), such polymer expansion converges for sufficiently low temperatures.

In order to do that, let us put the system in a finite box $\Lambda \subset \mathbb{Z}^{d}$ and let us define, for a fixed spin configuration $\sigma_{\Lambda}$ in $\Lambda$, the subset of $\Lambda$ given by $P=\left\{x \in \Lambda: \sigma_{x} \neq 0\right\}$. We view this set as the union of its connected components, i.e. $P=\cup_{i=1}^{n} p_{i}$ with each set $p_{i} \subset \Lambda$ being connected in the sense that for each partition $A, B$ of $p_{i}$ (i.e. $A \cup B=p_{i}$ and $A \cap B=\emptyset$ ) there exist $x \in A$ and $y \in B$ such that $|x-y|=1$. The configuration $\sigma_{\Lambda}$ induces a (non zero) spin configuration $s_{p_{i}}$ on each connected component $p_{i}$ of $P$ which is a function $s_{p_{i}}: p_{i} \rightarrow\{-1,+1\}: x \mapsto s_{x}$. The pairs $\boldsymbol{p}_{i}=\left(p_{i}, s_{p_{i}}\right)$ are the polymers associated to the configuration $\sigma_{\Lambda}$. By construction the correspondence $\sigma_{\Lambda} \leftrightarrow\left\{\boldsymbol{p}_{1}, \ldots, \boldsymbol{p}_{n}\right\}$ is one to one. The distance between two polymers $\boldsymbol{p}=\left(p, s_{p}\right)$ and $\tilde{\boldsymbol{p}}=\left(\tilde{p}, s_{\tilde{p}}\right)$ is the number $d(p, \tilde{p})=\min _{x \in p, y \in \tilde{p}}|x-y|$. Note that if $\left\{\boldsymbol{p}_{1}, \ldots, \boldsymbol{p}_{n}\right\}$ are the polymers associated to the configuration $\sigma_{\Lambda}$, then necessarily $d\left(p_{i}, p_{j}\right) \geq 2$ for all $\{i, j\} \subset\{1, \ldots, n\}$.

With these definitions we can rewrite the Hamiltonian of the system in a box $\Lambda \subset \mathbb{Z}^{d}$ with free boundary conditions as (here below $\beta$ is the inverse temperature)

$$
\beta H_{\Lambda}(\sigma)=\sum_{1 \leq i<j \leq n} W\left(\boldsymbol{p}_{i}, \boldsymbol{p}_{j}\right)+\sum_{i=1}^{n}\left[\beta D\left|p_{i}\right|-A\left(\boldsymbol{p}_{i}\right)\right]
$$

where

$$
\begin{gathered}
W\left(\boldsymbol{p}_{i}, \boldsymbol{p}_{j}\right)=-\beta \sum_{\substack{x \in p_{i} \\
y \in p_{j}}}\left[J_{x y} s_{x} s_{y}+K_{x y}\right] \\
A\left(\boldsymbol{p}_{i}\right)=\beta \sum_{\{x, y\} \subset p_{i}}\left[J_{x y} s_{x} s_{y}+K_{x y}\right]
\end{gathered}
$$

Observe now that to sum over configuration $\sigma_{\Lambda}$ in $\Lambda$ is equivalent to sum over polymers configurations $\left\{\boldsymbol{p}_{1}, \ldots, \boldsymbol{p}_{n}\right\}$ in $\Lambda$ such that $n \geq 0$ ( $n=0$, i.e. no polymers, is the ground state configuration) 
and $d\left(p_{i}, p_{j}\right) \geq 2$ for all pairs $\{i, j\} \subset\{1, \ldots, n\}$. Hence the partition function of the system, at inverse temperature $\beta$ and with free boundary conditions, is rewritten as

$$
\begin{aligned}
Z_{\Lambda}(\beta) & =\sum_{\sigma_{\Lambda}} e^{-\beta H\left(\sigma_{\Lambda}\right)} \\
& =1+\sum_{n \geq 1} \frac{1}{n !} \sum_{\substack{\left(\boldsymbol{p}_{1}, \ldots, \boldsymbol{p}_{n}\right) \in \mathcal{P}_{\Lambda}^{n} \\
d\left(p_{i}, p_{j}\right) \geq 2}} \rho_{\boldsymbol{p}_{1}} \ldots \rho_{\boldsymbol{p}_{n}} e^{-\sum_{i<j} W\left(\boldsymbol{p}_{i}, \boldsymbol{p}_{j}\right)}
\end{aligned}
$$

where

$$
\rho_{\boldsymbol{p}}=e^{-[\beta D|p|-A(\boldsymbol{p})]}
$$

and

$$
\mathcal{P}_{\Lambda}=\left\{\boldsymbol{p}=\left(p, s_{p}\right): p \subset \Lambda \text { connected, } s_{p} \text { function from } p \text { to }\{-1,+1\}\right\}
$$

We now extend the definition of $W\left(\boldsymbol{p}_{i}, \boldsymbol{p}_{j}\right)$ to all pairs in $\mathcal{P}$ as

$$
W\left(\boldsymbol{p}_{i}, \boldsymbol{p}_{j}\right)= \begin{cases}-\beta \sum_{\substack{x \in p_{i} \\ y \in p_{j}}}\left[J_{x y} s_{x} s_{y}+K_{x y}\right] & \text { if } d\left(p_{i}, p_{j}\right) \geq 2 \\ +\infty & \text { otherwise }\end{cases}
$$

With these definitions it is immediate to see that r.h.s. of (4.10) can be written as

$$
Z_{\Lambda}(\beta)=1+\sum_{n \geq 1} \frac{1}{n !} \sum_{\left(\boldsymbol{p}_{1}, \ldots, \boldsymbol{p}_{n}\right) \in \mathcal{P}_{\Lambda}^{n}} \rho_{\boldsymbol{p}_{1}} \ldots \rho_{\boldsymbol{p}_{n}} e^{-\sum_{1 \leq i<j \leq n} W\left(\boldsymbol{p}_{i}, \boldsymbol{p}_{j}\right)}
$$

which is the partition function of a polymer gas of the type (2.2) in which the polymers are elements of the set $\mathcal{P}$ defined by

$$
\mathcal{P}=\left\{\boldsymbol{p}=\left(p, s_{p}\right): p \subset \mathbb{Z}^{d} \text { connected and finite, } s_{p} \text { function from } p \text { to }\{-1,+1\}\right\}
$$

with activity given in (4.11) and with incompatibility relation $\boldsymbol{p} \nsim \tilde{\boldsymbol{p}} \Leftrightarrow d(p, \tilde{p})<2$. This pair interaction $W\left(\boldsymbol{p}_{i}, \boldsymbol{p}_{j}\right)$ is stable in the sense of (2.3). As a matter of fact it is easy to check that, for all $n \in \mathbb{N}$ and all $\left(\gamma_{1}, \ldots, \gamma_{n}\right) \in \mathcal{P}^{n}$.

$$
\sum_{1 \leq i<j \leq n} W\left(\boldsymbol{p}_{i}, \boldsymbol{p}_{j}\right) \geq-\sum_{i=1}^{n} B\left(\boldsymbol{p}_{i}\right)
$$

with

$$
B\left(\boldsymbol{p}_{i}\right)=\beta J\left|p_{i}\right|-A\left(\boldsymbol{p}_{i}\right)
$$

where $J$ is defined in (4.2) and $A\left(\boldsymbol{p}_{i}\right)$ is defined in (4.8). Again note that we have to check this condition on non intersecting sets of polymers since when $\left(\gamma_{1}, \ldots, \gamma_{n}\right)$ contains one o more incompatible pairs this inequality is trivially satisfied.

So, by theorem 1, the pressure of this polymer gas (i.e. the free energy of our long range BEG model) is absolutely convergent if there exist $\mu_{\boldsymbol{p}}$ such that such that

$$
e^{-\beta(D-J)|p|} \leq \mu_{\boldsymbol{p}} e^{-\sum_{\tilde{\boldsymbol{p}} \in \mathcal{P}} F(\boldsymbol{p}, \tilde{\boldsymbol{p}}) \mu_{\tilde{\boldsymbol{p}}}}, \quad \forall \gamma \in \mathcal{P}
$$


We choose

$$
\mu_{\boldsymbol{p}}=e^{-\beta(D-J)|p|} e^{\alpha|p|}
$$

Hence, inserting (4.16) in (4.15), we obtain that the pressure of such contour gas can be written in terms of an absolutely convergent series if, for some $\alpha>0$

$$
\sum_{\tilde{\boldsymbol{p}} \in \mathcal{P}} F(\boldsymbol{p}, \tilde{\boldsymbol{p}}) \mu_{\tilde{\boldsymbol{p}}} \leq \alpha|p|
$$

By bounding again $F(\boldsymbol{p}, \tilde{\boldsymbol{p}}) \leq 1$ whenever $\tilde{\boldsymbol{p}} \nsim \boldsymbol{p}$ (recall that the short range potential $U$ is in this case purely hard core), we get

$$
\begin{gathered}
\sum_{\tilde{\boldsymbol{p}} \in \mathcal{P}} F(\boldsymbol{p}, \tilde{\boldsymbol{p}}) \mu_{\tilde{\boldsymbol{p}}}=\sum_{\substack{\tilde{\boldsymbol{p}} \in \mathcal{P} \\
d(p, \tilde{p}) \leq 1}} \mu_{\tilde{\boldsymbol{p}}}+\sum_{\substack{\tilde{\boldsymbol{p}} \in \mathcal{P} \\
d(p, \tilde{p})>1}}|W(\boldsymbol{p}, \tilde{\boldsymbol{p}})| \mu_{\tilde{\boldsymbol{p}}} \leq \\
\quad \leq|p|\left[2 d \sup _{x \in \mathbb{Z}^{d}} \sum_{\substack{\tilde{\boldsymbol{p}} \in \mathcal{P} \\
x \in \boldsymbol{p}}} \mu_{\tilde{\boldsymbol{p}}}\right]+\max _{x \in p} \sum_{\substack{\tilde{\boldsymbol{p}} \in \mathcal{P} \\
d(x, \tilde{p})>1}}|W(\boldsymbol{p}, \tilde{\boldsymbol{p}})| \mu_{\tilde{\boldsymbol{p}}}
\end{gathered}
$$

where $d(x, \tilde{p})=\min _{y \in \tilde{p}}|x-y|$. Observe now that, by (4.4), $|W(\boldsymbol{p}, \tilde{\boldsymbol{p}})| \leq \beta J_{1}|p||\tilde{p}| n^{-(d+\lambda)}$ whenever $d(p, \tilde{p})=n$. Therefore

$$
\begin{aligned}
& \max _{x \in p} \sum_{\substack{\tilde{p} \in \mathcal{P} \\
d(\tilde{\boldsymbol{p}}, x)>1}}|W(\boldsymbol{p}, \tilde{\boldsymbol{p}})| \mu_{\tilde{\boldsymbol{p}}} \leq|p| \sum_{n>1} \frac{\beta J_{1}}{n^{d+\lambda}} \max _{x \in p} \sum_{\substack{\tilde{\boldsymbol{p}} \in \mathcal{P} \\
d(\tilde{p}, x)=n}}|\tilde{p}| \mu_{\tilde{\boldsymbol{p}}} \leq
\end{aligned}
$$

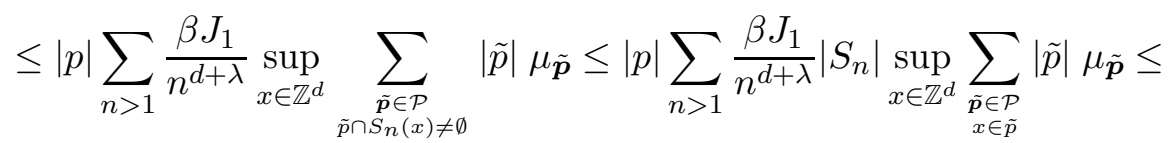

where $S_{n}=\left\{y \in \mathbb{Z}^{d}:|y|=n\right\}$. An easy calculation show that

$$
\left|S_{n}\right| \leq \frac{(2 d)^{d}}{d !} n^{d-1}
$$

So we get

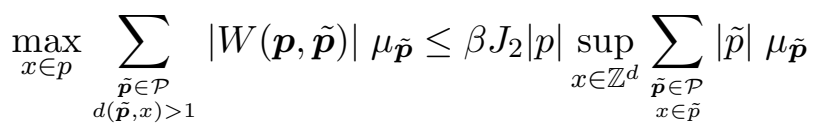

where

$$
J_{2}=\frac{(2 d)^{d} J_{1}}{d !} \sum_{n=2}^{\infty} \frac{1}{n^{1+\lambda}}
$$

Hence

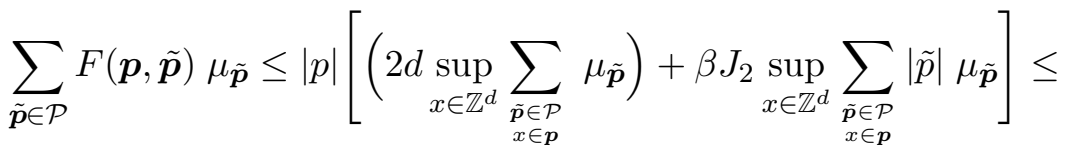

$$
\begin{aligned}
& \leq|p|\left[2 d+\beta J_{2}\right] \sup _{\substack { x \in \mathbb{Z}^{d} \\
\begin{subarray}{c}{\tilde{\boldsymbol{p}} \in \mathcal{P} \\
x \in \tilde{\boldsymbol{p}}{ x \in \mathbb { Z } ^ { d } \\
\begin{subarray} { c } { \tilde { \boldsymbol { p } } \in \mathcal { P } \\
x \in \tilde { \boldsymbol { p } } } }\end{subarray}}|\tilde{p}| \mu_{\tilde{\boldsymbol{p}}} \leq J_{\beta}|p| \sum_{\substack{\tilde{\boldsymbol{p}} \in \mathcal{P} \\
x \in \tilde{\boldsymbol{p}}}}|\tilde{p}| \mu_{\tilde{\boldsymbol{p}}}
\end{aligned}
$$


where

$$
J_{\beta}=2 d+\beta J_{2}
$$

Therfore, recalling (4.16), convergence condition (4.17) becomes

$$
J_{\beta} \sum_{n=1}^{\infty} n\left[e^{-\beta(D-J)} e^{\alpha}\right]^{n} 2^{n} C_{n} \leq \alpha
$$

where $C_{n}$ is the number of connected sets of vertices of $\mathbb{Z}^{d}$ with cardinality $n$ containing the origin (the factor $2^{n}$ in l.h.s. of (4.18) counts the number of functions from $p$ to $\{-1,+1\}$ when $|p|=n$ ). $C_{n}$ can be easily bounded by $C^{n}$ for some $C$, e.g. one can take $C_{n} \leq(4 d)^{n}$. So condition (4.18) becomes

$$
\sum_{n=1}^{\infty} n\left(x e^{\alpha}\right)^{n} \leq \frac{\alpha}{J_{\beta}}
$$

where

$$
x=8 d e^{-\beta(D-J)}
$$

Formulas (4.19) and (4.20) imply that

$$
e^{-\beta(D-J)} \leq\left[e^{-\alpha} f\left(\alpha / J_{\beta}\right)\right] \frac{1}{8 d}
$$

where

$$
f(u)=\frac{2 u}{2 u+1+\sqrt{4 u+1}}
$$

For example, taking $\alpha=1 / 2$ and bounding $f(u) \leq 2 u /(2 u+1)$ (we are not looking here for optimal estimates), we obtain that convergence occurs if

$$
e^{-\beta(D-J)} \leq \frac{1}{8 d \sqrt{e}\left(2 d+1+\beta J_{2}\right)}
$$

i.e., for all inverse temperatures $\beta \geq \beta_{0}$, where $\beta_{0}$ is the positive solution of the equation

$$
\left(2 d+1+\beta J_{2}\right)=\frac{e^{\beta(D-J)}}{8 \sqrt{e} d}
$$

\section{Acknowledgements.}

This work was supported by CNPq (Conselho Nacional de Desenvolvimento Científico e Tecnológico), a Brazilian Governmental agency promoting scientific and technology development.

\section{References}

[1] Battle, G. A. and Federbush, P.: A phase cell cluster expansion for euclidean field theory, Ann. of Phys. 142 (1982), 95-139. 
[2] Blume M., Emery, V. J. and Griffiths, R. B.: Ising Model for the $\lambda$ Transition and Phase Separation in He3-He4 Mixtures, Phys. Rev. A 4 (1971), 1071 - 1077

[3] Brydges, D. and Federbush, P.: A new form of the Mayer expansion in classical statistical mechanics, J. Math Phys. 19 (1978), 2064-2067.

[4] Brydges, D.: A Short Course on Cluster Expansion, Les Houches 1984, K. Osterwalder, R. Stora eds., North Holland Press (1986)

[5] Cammarota, C.: Decay of correlations for Infinite range Interactions in unbounded spin systems, Comm. Math Phys. 85 (1982), 517528.

[6] Dobrushin, R. L.: Estimates of semi-invariants for the Ising model at low temperatures. In "Topics in Statistical and Theoretical Physics", American Mathematical Society Translations, Ser. 2, 1775981 (1996).

[7] Dinaburg, E.I. and Sinai, Ya.G.: Contour Models with Interaction and their Applications, Sel. Math. Sov. 7 (1988), 291-315.

[8] Fernandez, R. and Procacci, A.: Cluster expansion for abstract polymer models. New bounds from an old approach, Preprint, arxiv math-ph/0605041.

[9] Gruber, C. and Kunz, H.: General properties of polymer systems. Comm. Math. Phys. 22 (1971), 133-61.

[10] Kotecký, R.and Preiss, D.: Cluster expansion for abstract polymer models. Comm. Math. Phys., 103 (1986), 491-498.

[11] Imbrie, J. Z.: Decay of correlations in the one dimensional Ising model with $J_{i j}=|i-j|^{-2}$ decay of correlations, Comm. Math. Phys. 85 (1982), 491-515.

[12] Miracle-Solé, S.: On the convergence of cluster expansions, Physica A 279 (2000), 244-249.

[13] Park, Y. M.: Extension of Pirogov-Sinai theory of phase transitions to infinite range interactions. I. Cluster expansion Comm. Math. Phys. 114(1988), 187-218.

[14] O. Penrose: Convergence of fugacity expansions for classical systems. In Statistical mechanics: foundations and applications, A. Bak (ed.), Benjamin, New York (1967).

[15] Pfister, Ch.E.: Large deviation and phase separation in the two-dimensional Ising model, Helv. Phys. Acta 64(1991), 953-1054.

[16] Procacci, A.: Cluster expansion methods in rigorous statistical mechanics, preprint, (www.mat.ufmg.br/aldo/papers/book.pdf).

[17] Procacci, A., de Lima, B. N. B. and Scoppola, B.: A Remark on high temperature polymer expansion for lattice systems with infinite range pair interactions, Lett. Math. Phys. 45 (1998), 303322 .

[18] Seiler, E.: Gauge Theories as a Problem of Constructive Quantum Field Theory and Statistical Mechanics, Lecture Notes in Physics 159, Springer-Verlag, BerlinHeidelberg New York (1982). 
[19] Sokal, A.: Bounds on the complex zeros of (di)chromatic polynomials and Potts-model partition functions. Combin. Probab. Comput. 10 (2001), 41-77.

[20] Ueltschi, D.: Cluster expansions and correlation functions, Moscow Math. J. 4 (2004), 511-522. 